\title{
Intelligent systems for future generation communications
}

\author{
Deok Gyu Lee • Sajid Hussain • \\ Alan Chin-Chen Chang
}

Published online: 21 April 2010

(C) Springer Science+Business Media, LLC 2010

The ever-growing Future Generation Communications (FGC) provides an Intelligent and Ubiquitous Communication and Network Technology for Tomorrow. That is, the FGC has emerged rapidly as an exciting new paradigm that includes ubiquitous, grid, and peer-to-peer computing to provide computing and communication services at any time and anywhere. In order to realize the advantages of such services, it is important that intelligent systems be suitable for FGC. This special issue aims to foster the dissemination of high quality research in any new FGC idea, method, theory, technique, and research.

This special issue brings together seven research papers providing a snapshot of the current state of the art of intelligent systems for FGC. Many high-quality papers were received and only seven papers were finally selected after a rigorous review process by international experts to be published in this issue.

In "Design and Implementation of a Framework for Building Distributed Smart Object Systems", Fahim Kawsar, et al. propose a document-oriented framework to support development of human-centered intelligent systems for pervasive environment. In the framework, applications' requirements and component services are objectified by structured documents and a runtime infrastructure provides the pair-wise

D.G. Lee $(\varangle)$

ETRI, Daejeon, Korea

e-mail: deokgyulee@gmail.com

S. Hussain

Fisk University, Nashville, USA

e-mail: dr.sajid.hussain@gmail.com

A.C.-C. Chang

National Chung Cheng University, Minxiong, Taiwan

e-mail: alan3c@gmail.com 
mapping using structural type matching thus externalizing component management and addressing heterogeneity issues away from the applications making application development very simple and rapid.

The paper "HMM Machine Learning and Inference for Activities of Daily Living Recognition" by Bo-Chao Cheng, et al. focus on the design of the inference engine to recognize Activities of Daily Living (ADL), defined as a measurement of the functional status of a person in a ubiquitous computing system. A good ADL recognition engine should be able to adjust its inference strategy based on the reasoning ability to learn in order to avoid a high error rate in real world applications. The authors proposed an efficient inference engine, called the Adaptive Learning Hidden Markov Model (ALHMM), which provides a comprehensive recognition capability with an effective learning process.

In "A Context-aware Multi-Model Remote Controller for Electronic Home Devices", Chin-Feng Lai, et al. propose and instantiate the context-aware multi-mode remote controller for electronic home devices to relieve user off nuisance of enormous remote controllers. The smart heterogeneous network control platform is developed to provide a shared access control mechanism, generates a simple user interface from the control function and information of the current devices. By the way, in order to recommend the personalized service on the remote controller, the low complexity computing algorithm is used based on the previous usage habits.

The paper "u-BabSang: A Context-aware Food Recommendation System" by Yoosoo, et al. deals with the problem of recommending appropriate foods for each individual's health at the table in real time. The proposed system recommends foods by integrating the multiple contexts obtained from the sensors that represent a user's profile, physiological signals, and environmental information around the dining table. The authors present a context-aware food recommendation system that provides individualized food recommendation lists at the dining table, and is based dietary advice in the typical Korean medical text.

In "Privacy-Enhanced Key Recovery in Mobile Communication Environments," Jong Hyuk Park proposes a new security-enhanced key recovery (KR) protocol and constructs a privacy-enhanced KR protocol, which is based on the security-enhanced KR protocol for protecting users' location privacy.

The paper "Context-aware Cross Layer Optimized Video Streaming in Wireless Multimedia Sensor Networks" by Lei, et al. deals with the problem of video streaming gathering in wireless multimedia sensor networks. The authors present a new cross-layer optimized scheme based on the carefully designed TPGF multipath routing protocol and gathered context information by real sensors. This research effort is the first work that integrates the concept of context-awareness with cross layer optimization for facilitating multimedia streaming in WMSNs.

The paper "A Power-, Delay- and Emergency-Efficient Protocol of Ubiquitous Sensor Network Systems for Silver Town Applications" by YunSeop Yu, et al. is presented. For this protocol, a fixed node is established to one point which can communicate with one-hop to both the sink node and the mobile sensor nodes in the dark region. The fixed nodes are operated like the fixed cluster head. The simulated life time of the proposed routing algorithm is longer than those of LEACH and HEED and the simulated time delay of the proposed routing algorithm is faster than those 
of LEACH and HEED when the three protocols are applied to a specific silver town environment.

Finally, we strongly believe that the selected papers make a significant contribution to researchers, practitioners, and students working in the areas of intelligent systems for future generation communications. We would like to express our sincere appreciation to all the authors for their valuable contributions and also to the referees for their cooperation and hard work in reviewing the papers in a timely and professional manner. Our special thanks go to the editorial board for this SI and Prof. Hamid R. Arabnia who is the Editor in Chief of this journal, for his support throughout the whole publication processes. 